\title{
A brief perspective on the diverging theories of lymphatic targeting with colloids
}

\author{
This article was published in the following Dove Press journal: \\ International Journal of Nanomedicine \\ 15 June 2016 \\ Number of times this article has been viewed
}

\author{
Karthik Siram' \\ Gregory Marslin² \\ Chellan Vijaya Raghavan' \\ Krishnamoorthy Balakumar ${ }^{\prime}$ \\ Habibur Rahman' \\ Gregory Franklin ${ }^{3}$ \\ 'Department of Pharmaceutics, PSG \\ College of Pharmacy, Coimbatore, \\ India; ${ }^{2}$ Centre for the Research and \\ Technology of Agro-Environment \\ and Biological Sciences, University of \\ Minho, Braga, Portugal; ${ }^{3}$ Department \\ of Integrative Plant Biology, Institute \\ of Plant Genetics, Polish Academy of \\ Sciences, Poznań, Poland
}

Correspondence: Chellan Vijaya

Raghavan

Department of Pharmaceutics, PSG

College of Pharmacy, Peelamedu,

Coimbatore, India 64I004

$\mathrm{Tel}+9|9843| 28373$

Email drvijayaragha@gmail.com

Gregory Franklin

Department of Integrative Plant Biology, Institute of Plant Genetics, Polish

Academy of Sciences, Strzeszyńska 34,

60-479 Poznań, Poland

Tel +48 6I 6550266

$\mathrm{Fax}+48616550301$

Email fgre@igr.poznan.pl

\begin{abstract}
For targeted delivery of colloids to the lymphatic system, the colloids should efficiently reach and remain in the lymphatics for a considerable period of time. As per the current knowledge, diffusion and phagocytosis are the two mechanisms through which colloids reach the lymphatic system. Several parameters including particle size and charge have been shown to affect the direct uptake of colloids by the lymphatic system. Although many researchers attached ligands on the surface of colloids to promote phagocytosis-mediated lymphatic delivery, another school of thought suggests avoidance of phagocytosis by use of carriers like polyethylene glycol (PEG)ylated colloids to impart stealth attributes and evade phagocytosis. In this perspective, we weigh up the paradoxical theories and approaches available in the literature to draw conclusions on the conditions favorable for achieving efficient lymphatic targeting of colloids.
\end{abstract}

Keywords: lymphatic targeting, colloids, PEGylation, phagocytosis

\section{Introduction}

The lymphatic system (lymphatics), which encompasses the circulating lymph, network of lymphatic pathways (lymphatic capillaries, collecting vessels, trunks, and ducts), and lymphatic organs (lymph nodes, thymus, bone marrow, tonsils, spleen, etc) is present throughout the body in conjunction with the systemic circulation. The lymph is formed by the transport of interstitial fluid surrounding the blood vessels into the lymphatic capillaries. Thus, the lipids, enzymes, and protein composition of lymph and plasma remain the same and vary only in concentrations. ${ }^{1,2}$ The lymphatic system removes foreign bodies from the body and maintains homeostasis. Apart from mediating the immune functions and tissue fluid balance, the lymphatics also act as a reservoir for human immunodeficiency virus, filariasis, tuberculosis, and metastatic cancer cells. ${ }^{3}$ Hence, lymphatic targeting of molecules, compounds, vaccines, and so on will be useful for diagnosis and therapy and eliciting immune responses. Colloids have emerged as an important class of drug carriers for targeted delivery into the complex lymphatic system. ${ }^{4}$ Targeting colloids to the lymphatics involves two major steps: first, the targeting material should reach the lymphatic structures and then it should reside there for a considerable period of time by evading the host immune trafficking mechanisms. A plethora of articles on lymphatic targeting using colloids suggests that several parameters such as surface charge, particle size, molecular weight, route of administration, nature of the colloid and its surface properties (hydrophilicity and lipophilicity), and so on play a role in targeting colloids to the lymphatics, and a discussion on each of those factors is out of focus of this perspective as it would be a mere repetition of the already established facts. However, the role of phagocytosis and polyethylene glycol (PEG) attachment to colloids (PEGylation) on lymphatic 
targeting is not clear. Logically, phagocytosis (an innate protective mechanism through which the body disposes all the antigens including colloids) and PEGylation (a strategy that imparts covertness with respect to phagocytosis) are two contrasting strategies, which cannot go hand in hand. Since the earlier issues have so far not been brought into the light, we intend to discuss for the first time those conflicting theories to derive a universal strategy for lymphatic targeting in this article.

\section{Charge: a driving force?}

Oussoren and Storm ${ }^{5}$ reported that surface charge of colloids does not play a role in lymphatic targeting. Nevertheless, many other studies have shown that particle surface charge is indeed an important factor for lymphatic delivery of colloids. $^{6-9}$ These studies argue that due to the repulsive drive exerted by the negatively charged interstitial matrix, negatively charged substances possess better lymphatic uptake than positive and neutral substances. Patel et $\mathrm{al}^{9}$ found that negatively charged colloidal particles had superior lymphatic uptake efficiency compared with positively charged particles, which in turn performed better than those with a neutral charge. In contrast, a few reports have stated that neutrally charged particles could more quickly enter the regional lymphatics after administration, whereas positively charged particles were better in terms of final uptake. ${ }^{10-12}$ A rapid clearance of negatively charged colloids when compared to neutral colloids has also been reported. ${ }^{13}$ Although the emerging depiction is that colloids can reach the lymphatics irrespective of their charge, their entry is possibly momentarily affected by factors such as the type of colloid used (eg, liposomes, polymeric nanoparticles, dendrimers, and solid lipid nanoparticles), biopharmaceutical factors, $\mathrm{pH}$, and net charge (due to ions) of the local interstitial fluid. But, the leads obtained relating to the host defense mechanisms that remove colloids from the body indicate that neutrally charged colloids hold an edge over positively or negatively charged colloids for lymphatic targeting.

\section{Phagocytosis: a dead end for colloids}

Phagocytosis is an important host immune defense mechanism performed by a kind of white blood cells known as macrophages. They are capable of scavenging both exogenous (viruses, bacteria, antigens, xenobiotics, nanoparticles, etc) and endogenous (free radicals, apoptotic and necrotic bodies, etc) danger signals from the body by a membraneengulfing, energy-consuming process called phagocytosis. The danger signals destined for phagocytosis are first marked with proteins called opsonins in a process termed opsonization. Immunoglobulins (IgG, IgM, IgD, etc), complement proteins (C3, C4, and C5), C-reactive protein, and so on are some of the opsonins that are adsorbed to the foreign bodies by Brownian motion and thereby mark them for phagocytosis. ${ }^{14}$ Finally, the phagocytosed material will be enzymatically broken down and removed from the body. Several researchers have attempted to exploit this natural mechanism to target lymphatics via attaching ligands to the colloids, as these ligands have the capacity to adsorb opsonins and prime the colloids for uptake by the macrophages. For example, ligands such as mannose, ${ }^{8}$ galactose, ${ }^{15} \mathrm{IgG},{ }^{16}$ and phosphatidylserine ${ }^{17,18}$ have been attached to colloids in order to promote phagocytosis. The theory behind this approach is that colloids bearing these ligands will be engulfed by macrophages in the interstitial spaces and subsequently be delivered into the lymphatics. Although this concept sounds persuasive, actually these colloids will likely be digested and eliminated from the body at a faster rate by the macrophages at the lymphatics. Hence, despite reaching the target, drugs carried by such colloids will not be therapeutically useful. Thus, using ligands to function as bait for attracting the macrophages would serve no use and will only lead to the rapid clearance of colloids from the body. On the other hand, this principle might be of great value for targeting leishmanial parasites residing in the macrophages and for the delivery of vaccines to elicit an immune response. ${ }^{19}$ Since lymphatic uptake of colloids has been shown to occur under conditions where phagocytosis is blocked, ${ }^{20,21}$ we conclude that phagocytosis might not play a role in lymphatic delivery of colloids.

\section{PEGylation: gateway for lymphatic targeting}

In sharp contrast to the earlier strategy, several researchers consider phagocytosis as an unfavorable phenomenon for the delivery of colloids into the lymphatic system. Hence, there were attempts to impart disguising properties to protect the colloids from phagocytosis. PEG is a nonionic surface-active polymer made of hydrophilic polyethylene oxide (PEO) and hydrophobic polypropylene oxide. Coating, attaching, or grafting of PEG polymer chains to a molecule or surface is called PEGylation. PEGylation imparts a stealth nature to the particles by forming a protective barricade against the adhesion of opsonins and thereby help them to evade phagocytosis. ${ }^{22}$ When opsonins adhere to the surface of PEGylated colloid, the confirmation of PEO units within PEG is disturbed, creating a force that resists opsonin adherence. 
As a result, a steric barrier is imparted to the colloid, which would enable the colloid to remain in the body for a longer time by evading phagocytosis. Furthermore, in addition to providing the hydrophilic surface required for lymphatic uptake, PEGylation also reduces the electrostatic interaction between the surface of the colloid and the components of the interstitial matrix by providing a neutral charge, which assists its drainage into the lymphatics. Thus, PEGylation has a positive impact not only in providing stealth attributes to colloids in blood ${ }^{23-26}$ but also in facilitating their drainage from the site of injection to the lymphatics.

The lymphatic uptake and retention of PEGylated colloids depend on the steric barrier induced by the number of PEO units. Although an increase in the number of PEO units increases the steric barrier and lymphatic uptake, a negative correlation exists between the steric barrier and lymph node retention. ${ }^{27}$ Hence, only a specific number of PEO units should be used for PEGylation to achieve good uptake into the lymphatics with increased residence time. Interestingly, inhibiting phagocytosis using dextran as an adjuvant increased both lymphatic uptake and residence time of PEGylated colloids. ${ }^{21}$ PEGylation can be applied in developing various nanoparticulate carriers like dendrimers, ${ }^{10}$ PRINT (particle replication in nonwetting templates) hydrogels, ${ }^{28}$ magnetic carbon nanotubes with PEG groups, ${ }^{29}$ solid lipid nanoparticles, ${ }^{3}$ polymeric nanoparticles, ${ }^{24}$ liposomes, ${ }^{25}$ and so on. Furthermore, a glance at a few of the promising nanopharmaceuticals in clinical use or undergoing trials like Oncaspar (PEGylated L-asperginase for acute lymphoblastic leukemia), Genexol (PEGylated block polymer containing an anticancer drug for metastatic breast cancer), and Lipo-dox and ThermoDox (PEGylated liposomal forms of doxorubicin) highlights the utility of PEGylation. ${ }^{30-32}$

\section{Size-dependent retention in lymphatics}

Although PEGylation imparts stealth characteristics facilitating the evasion of phagocytosis, retention of colloids in the lymphatics is largely dependent on their size. For instance, colloids in the size range of 10-100 nm can freely move through the interendothelial spaces of lymphatic capillaries irrespective of PEGylation. Studies have revealed that although PEGylation improved lymphatic uptake, it reduced the retention time of colloids. , $3,10,33$ The authors hypothesized that the poor retention of the colloids in the lymph nodes is due to the smaller size and extreme hydrophilic nature of PEGylated colloids. These colloids probably escaped through the spaces present between the lymphatic capillary cells because of their smaller size. The environment in the lymphatics is not much different from the systemic circulation, and the colloids will be immersed in an environment containing several kinds of immune cells (lymphocytes, macrophages, monocytes, etc), which would also potentially act to eliminate them from the lymphatics. It is also possible that the PEG coating will be eroded from the colloids over a period of time, which might eventually allow the adhesion of opsonins on the particles marking them for phagocytosis and subsequent destruction. If they escape from these immune cells, the colloids will pass through the lymphatic capillaries and lymph nodes to reach the systemic circulation. Since the lymph nodes are acting as simple mechanical filters, ${ }^{2}$ they can retain the colloids if the size is greater than $100 \mathrm{~nm}$. Hence, for extending the retention time of colloids in the lymphatics, their size should exceed this at the time of entry. On the other hand, to successfully evade phagocytosis in the lymphatics, the particle size should be less than $100 \mathrm{~nm}$. One possibility to achieve larger particle size in the lymphatics is to promote a mild cohesion among the particles. For example, a few neutrally charged particles in the range of 10-100 nm could possibly cohere to form a bigger particle due to the lack of surface attractive or repulsive forces. Theoretically, this concept sounds simple, but achieving this in vivo is confounded by the influence of several factors like lack of proper cohesion among sterically stabilized colloids, the microenvironment around the colloids, and physiological conditions of the lymphatics in diseased states, etc. Use of a nonionic stabilizer (possibly PEG) in the preparation of colloids might favor this mild cohesion, as anionic or cationic stabilizers exert a repulsive force among the colloids.

\section{Seeds and leads from filarial worms for lymphatic targeting}

Lymphatic filarial pathogenesis provides an insight into how the lymphatic system could be targeted by colloids. ${ }^{3,33}$ In spite of the surrounding immune trafficking cells and oxidative free radicals, filarial worms survive in the lymphatics for several years. These worms contain a thick cuticle comprising an internal matrix of collagenous proteins, which allow them to survive in the face of various host immune reactions. Moreover, their morphology facilitates the worms' survival in the lymphatics by protecting against macrophages and other immune cells. Worm-shaped colloidal particles were able to evade macrophages and extend the half-life of anticancer drugs in the body. ${ }^{34-36}$ According to the authors, the short cylindrical conformation of the wormlike structures prevents the interaction with macrophages and even if the macrophages interact 
Table I Snapshot of the effect of various properties of colloidal particles on lymphatic targeting

\begin{tabular}{lll}
\hline Property & $\begin{array}{l}\text { Lymphatic } \\
\text { uptake }\end{array}$ & $\begin{array}{l}\text { Lymphatic } \\
\text { retention }\end{array}$ \\
\hline Size less than $100 \mathrm{~nm}$ & + & + \\
Neutral charge & + & + \\
PEGylation & + & + \\
Phagocytosis & + & - \\
Worm shape & $*$ & ++ \\
\hline
\end{tabular}

Notes: +, promotes; ++, strongly promotes; -, does not promote; *insufficient data available.

with the worms, they are dragged off from the worm's surface due to the hydrodynamic shear exerted by the flow of blood. In addition, these worms secrete glutathione- $S$-transferases to counteract the oxidative free radicals produced by the host. ${ }^{37}$ Further studies on lymphatic targeting using glutathione$S$-transferases will reveal whether it might prove helpful in extending the occupancy time of colloids in lymphatics.

\section{Conclusion}

We have analyzed the contrasting reports about surface charge of particles, effect of PEGylation, and usage of phagocytosis for lymphatic targeting and residence. On the basis of this analysis, we predict that neutrally charged colloids, preferably PEGylated, with a size less than $100 \mathrm{~nm}$ will be efficiently taken up by the lymphatics (Table 1). We also refute the concept that promotion of phagocytosis by means of liganddecorated colloids is a useful strategy for lymphatic targeting (Figure 1). Promoting mild cohesion among colloids in the lymphatics to form larger particles would possibly increase the retention of colloids. Research focused on worm-shaped nanoparticles and the use of glutathione- $S$-transferases could provide further insights in the field of lymphatic targeting.

\section{Acknowledgments}

GF is supported by the EU FP7 BIOTALENT project (GA621321) and cofinanced by funds allocated for education

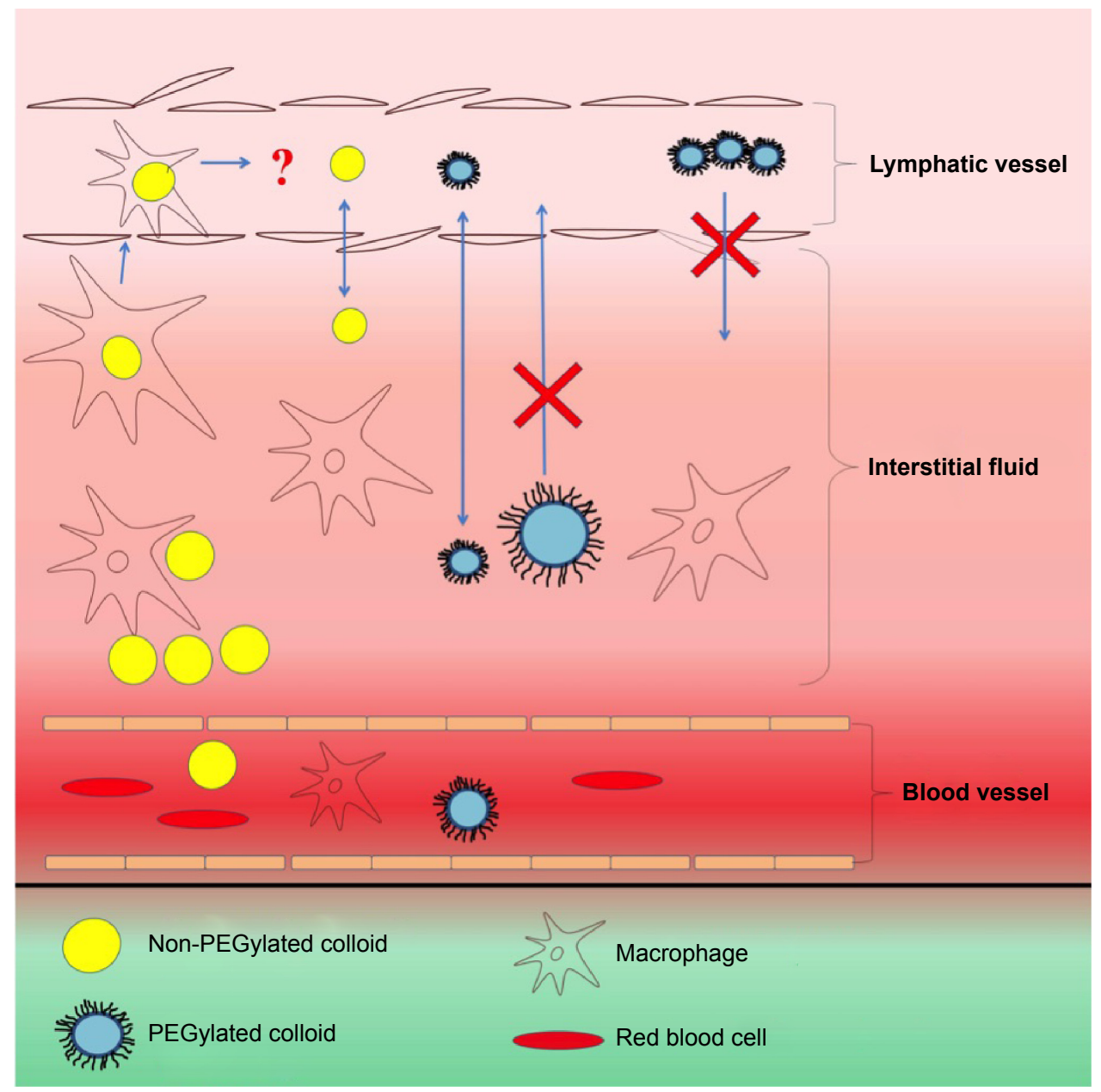

Figure I Schematic diagram of the kinetics of colloids in interstitial fluid. Notes: $\times$ denotes does not occur. ? denotes the fate remains skeptical. 
through project number W26/7.PR/2015 [GA 3413/7. PR/2015/2] for the years 2015-2019.

\section{Disclosure}

The authors report no conflicts of interest in this work.

\section{References}

1. Cho H-Y, Lee Y-B. Nano-sized drug delivery systems for lymphatic delivery. J Nanosci Nanotechnol. 2014;14(1):868-880.

2. Hawley AE, Davis SS, Illum L. Targeting of colloids to lymph nodes: Influence of lymphatic physiology and colloidal characteristics. $A d v$ Drug Deliv Rev. 1995;17(1):129-148.

3. Siram K, Chellan VR, Natarajan T, et al. Solid lipid nanoparticles of diethylcarbamazine citrate for enhanced delivery to the lymphatics: in vitro and in vivo evaluation. Expert Opin Drug Deliv. 2014;11(9): 1351-1365.

4. Sheeba CJ, Marslin G, Revina AM, Franklin G. Signaling pathways influencing tumor microenvironment and their exploitation for targeted drug delivery. Nanotechnol Rev. 2014;3(2):123-151.

5. Oussoren C, Storm G. Liposomes to target the lymphatics by subcutaneous administration. Adv Drug Deliv Rev. 2001;50(1-2):143-156.

6. Rao DA, Forrest ML, Alani AWG, Kwon GS, Robinson JR. Biodegradable PLGA based nanoparticles for sustained regional lymphatic drug delivery. J Pharm Sci. 2010;99(4):2018-2031.

7. Takakura Y, Atsumi R, Hashida M, Sezaki H. Development of a novel polymeric prodrug of mitomycin $\mathrm{C}$, mitomycin $\mathrm{C}$-dextran conjugate with anionic charge. II. Disposition and pharmacokinetics following intravenous and intramuscular administration. Int J Pharm. 1987; 37(1-2):145-154.

8. Kaur CD, Nahar M, Jain NK. Lymphatic targeting of zidovudine using surface-engineered liposomes. J Drug Target. 2008;16(10):798-805.

9. Patel HM, Boodle KM, Vaughan-Jones R. Assessment of the potential uses of liposomes for lymphoscintigraphy and lymphatic drug delivery. Failure of 99m-technetium marker to represent intact liposomes in lymph nodes. Biochim Biophys Acta. 1984;801(1):76-86.

10. Kaminskas LM, Kota J, McLeod VM, Kelly BD, Karellas P, Porter CJ. PEGylation of polylysine dendrimers improves absorption and lymphatic targeting following SC administration in rats. $J$ Control Release. 2009;140(2):108-116.

11. Kim CK, Lee MK, Han JH, Lee BJ. Pharmacokinetics and tissue distribution of methotrexate after intravenous injection of differently charged liposome-entrapped methotrexate to rats. Int $J$ Pharm. 1994;108(1):21-29.

12. Li SD, Huang L. Pharmacokinetics and biodistribution of nanoparticles. Mol Pharm. 2008;5(4):496-504.

13. Levchenko TS, Rammohan R, Lukyanov AN, Whiteman KR, Torchilin VP. Liposome clearance in mice: The effect of a separate and combined presence of surface charge and polymer coating. Int J Pharm. 2002;240(1-2):95-102.

14. Mortimer GM, Butcher NJ, Musumeci AW, Deng ZJ, Martin DJ, Minchin RF. Cryptic epitopes of albumin determine mononuclear phagocyte system clearance of nanomaterials. ACS Nano. 2014;8(4): 3357-3366.

15. Garg M, Jain NK. Reduced hematopoietic toxicity, enhanced cellular uptake and altered pharmacokinetics of azidothymidine loaded galactosylated liposomes. J Drug Target. 2006;14(1):1-11.

16. Mangat $S$, Patel HM. Lymph node localization of non-specific antibodycoated liposomes. Life Sci. 1985;36(20):1917-1925.

17. Oussoren C, Zuidema J, Crommelin DJA, Storm G. Lymphatic uptake and biodistribution of liposomes after subcutaneous injection. II. Influence of liposomal size, lipid composition and lipid dose. Biochim Biophys Acta. 1997;1328(2):261-272.
18. Wu MS, Robbins JC, Bugianesi RL, Ponpipom MM, Shen TY. Modified in vivo behavior of liposomes containing synthetic glycolipids. Biochim Biophys Acta. 1981;674(1):19-29.

19. Reddy ST, van der Vlies AJ, Simeoni E, et al. Exploiting lymphatic transport and complement activation in nanoparticle vaccines. Nat Biotechnol. 2007;25(10):1159-1164.

20. Oussoren C, Velinova M, Scherphof G, Van Der Want JJ, Van Rooijen N, Storm G. Lymphatic uptake and biodistribution of liposomes after subcutaneous injection IV. Fate of liposomes in regional lymph nodes. Biochim Biophys Acta. 1998;1370(2):259-272.

21. Feng L, Zhang L, Liu M, et al. Roles of dextrans on improving lymphatic drainage for liposomal drug delivery system. J Drug Target. 2010; 18(3):168-178.

22. Bailon P, Won C. PEG-modified biopharmaceuticals. Expert Opin Drug Deliv. 2009;6(1):1-16.

23. Biswas S, Dodwadkar NS, Deshpande PP, Parab S, Torchilin VP. Surface functionalization of doxorubicin-loaded liposomes with octaarginine for enhanced anticancer activity. Eur J Pharm Biopharm. 2013; 84(3):517-525.

24. Marslin G, Revina AM, Khandelwal VKM, Balakumar K, Sheeba CJ, Franklin G. PEGylated ofloxacin nanoparticles render strong antibacterial activity against many clinically important human pathogens. Colloids Surf B Biointerfaces. 2015;132:62-70.

25. Nakamura K, Yamashita K, Itoh Y, Yoshino K, Nozawa S, Kasukawa H. Comparative studies of polyethylene glycol-modified liposomes prepared using different PEG-modification methods. Biochim Biophys Acta. 2012;1818(11):2801-2807.

26. Owens DE, Peppas NA. Opsonization, biodistribution, and pharmacokinetics of polymeric nanoparticles. Int J Pharm. 2006;307(1):93-102.

27. Moghimi SM, Hawley AE, Christy NM, Gray T, Illum L, Davis SS Surface engineered nanospheres with enhanced drainage into lymphatics and uptake by macrophages of the regional lymph nodes. FEBS Lett. 1994;344(1):25-30.

28. Mueller SN, Tian S, DeSimone JM. Rapid and persistent delivery of antigen by lymph node targeting PRINT nanoparticle vaccine carrier to promote humoral immunity. Mol Pharm. 2015;12(5):1356-1365.

29. Yang F, Fu DL, Long J, Ni QX. Magnetic lymphatic targeting drug delivery system using carbon nanotubes. Med Hypotheses. 2008;70(4): 765-767.

30. Chang HI, Yeh MK. Clinical development of liposome-based drugs: formulation, characterization, and therapeutic efficacy. Int J Nanomedicine. 2012;7:49-60.

31. Weissig V, Pettinger T, Murdock N. Nanopharmaceuticals (part 1): products on the market. Int J Nanomedicine. 2014;9:4357-4373.

32. Sanna V, Pala N, Sechi M. Targeted therapy using nanotechnology: focus on cancer. Int J Nanomedicine. 2014;9(1):467-483.

33. Ali M, Afzal M, Bhattacharya SM, Ahmad FJ, Dinda AK. Nanopharmaceuticals to target antifilarials: a comprehensive review. Expert Opin Drug Deliv. 2013;10(5):665-678.

34. Geng Y, Dalhaimer P, Cai S, et al. Shape effects of filaments versus spherical particles in flow and drug delivery. Nat Nanotechnol. 2007;2(4): 249-255.

35. Kamaly N, Xiao Z, Valencia PM, Radovic-Moreno AF, Farokhzad OC. Targeted polymeric therapeutic nanoparticles: design, development and clinical translation. Chem Soc Rev. 2012;41(7):2971-3010.

36. Schroeder A, Heller DA, Winslow MM, et al. Treating metastatic cancer with nanotechnology. 2012. Nat Rev Cancer. 2011;12(1):39-50.

37. Gupta S, Rathaur S. Filarial glutathione S-transferase: its induction by xenobiotics and potential as drug target. Acta Biochim Pol. 2005;52(2): 493-500. 
International Journal of Nanomedicine

Dovepress

\section{Publish your work in this journal}

The International Journal of Nanomedicine is an international, peerreviewed journal focusing on the application of nanotechnology in diagnostics, therapeutics, and drug delivery systems throughout the biomedical field. This journal is indexed on PubMed Central,

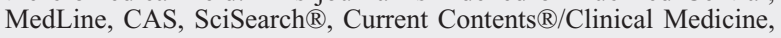

Journal Citation Reports/Science Edition, EMBase, Scopus and the Elsevier Bibliographic databases. The manuscript management system is completely online and includes a very quick and fair peer-review system, which is all easy to use. Visit http://www.dovepress.com/ testimonials.php to read real quotes from published authors.

Submit your manuscript here: http://www.dovepress.com/international-journal-of-nanomedicine-journal 